\title{
Ciudad Educadora
}

\section{he ahí el problema}

$\frac{\text { 흘 }}{\text { 응 }}$

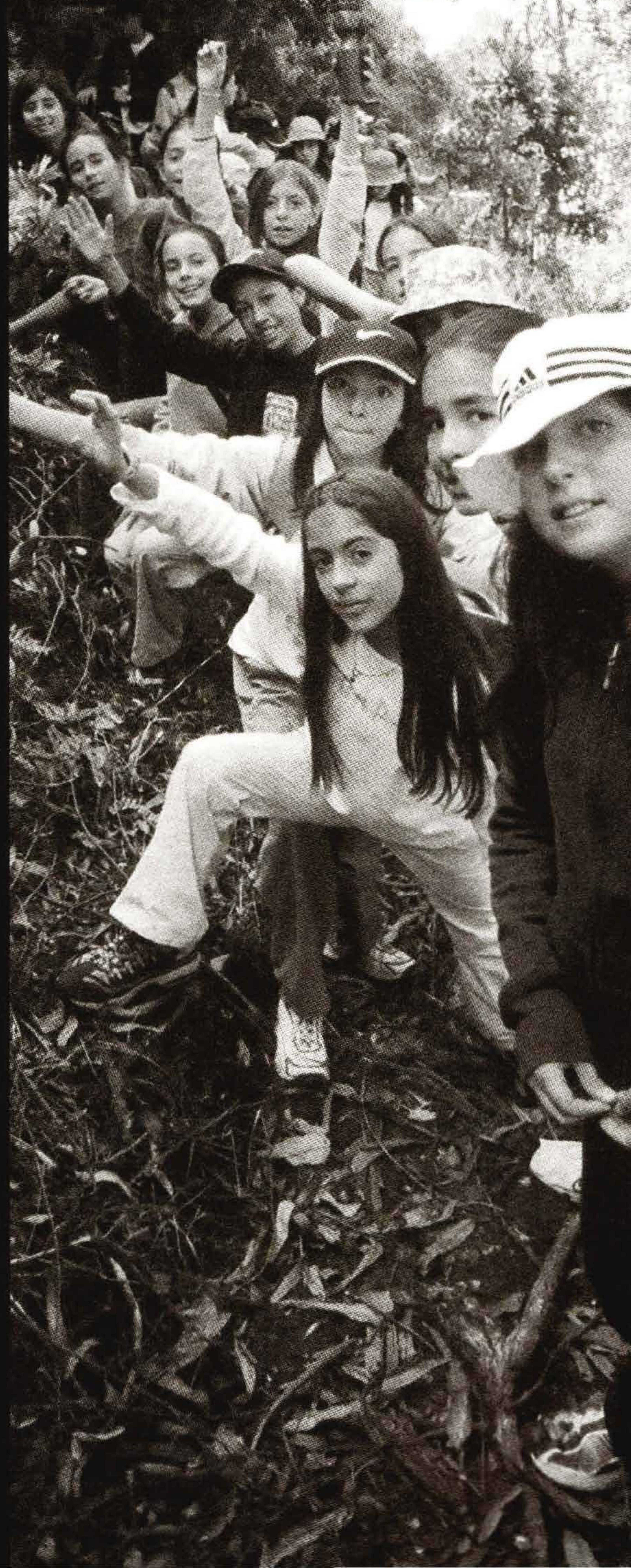




\section{Jorge Orlando Castro Villarraga}

Magíster en Sociología de la Universidad Nacional de Colombia y licenciado en Pedagogía y Psicología de la Universidad Pedagógica Nacional. Investigador y consultor educativo. Codirector del Seminario de Coordinadores Cátedra de Pedagogía 2005.

\section{Resumen}

Este artículo da cuenta de los propósitos y de algunos aportes conceptuales generados en el Seminario de coordinadores de la Cátedra de Pedagogía 2005 en torno a la idea y al modelo de Ciudad Educadora. Recoge las discusiones generadas a través de los protocolos y en las ponencias presentadas en la modalidad de simposio por sus integrantes, inscritas según tres directrices temáticas.

\section{Palabras clave}

Cátedra de Pedagogía, Ciudad Educadora, Escuela, Bogotá.

\section{Abstract}

This article summarizes both the purposes and some of the conceptual contributions from the Coordinator Seminar of the Cátedra de Pedagogía 2005 whose topic was the Educating City. The article contains the debates brought up by the protocols and conferences presented during the Simposium in three great thematic vertices.

\section{Key words}

Cátedra de Pedagogía, Educating City, School, Bogotá. 

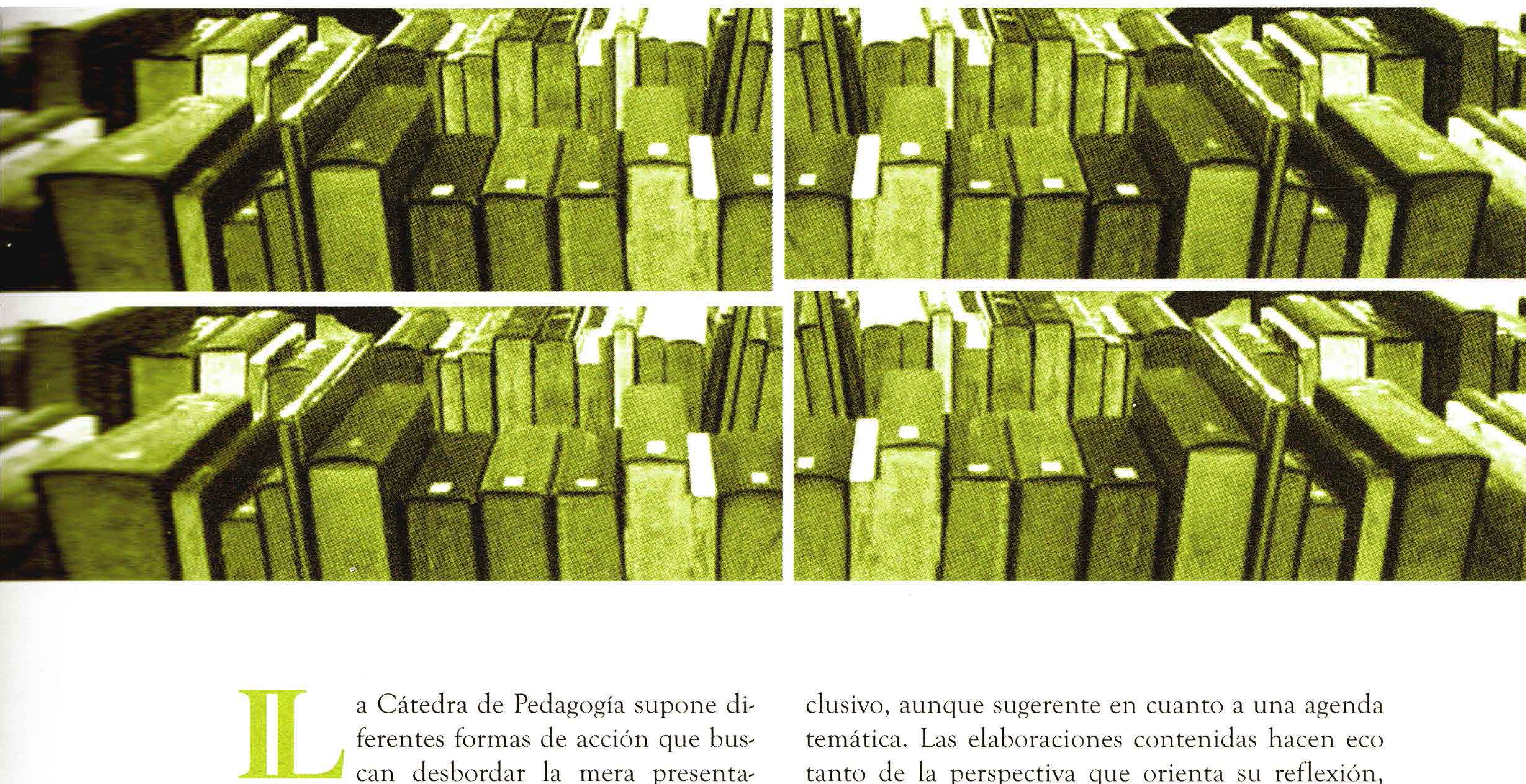

a Cátedra de Pedagogía supone diferentes formas de acción que buscan desbordar la mera presentación magistral. Esto quiere decir que la cátedra es más que cátedra, toda vez que incluye modalidades de trabajo, de encuentro, como conferencias magistrales, seminarios, simposios, talleres, jornadas pedagógicas, recorridos y reconocimiento. La cátedra, vista en su conjunto, es una opción por el descentramiento de la palabra, de la escritura, de la discusión, por poner nociones en movimiento, en interlocución con maestros, profesores, académicos, directivos, niños y padres, comunidades, grupos y redes. Este texto busca mostrar la discusión generada en una de estas modalidades, el Seminario de coordinadores, parte integrante de la Cátedra de Pedagogía 2005, desde la cual se trazaron algunas directrices temáticas para agrupar las discusiones y aportes conceptuales relacionados con la idea y el modelo de Ciudad Educadora. Se trata entonces de mostrar un proceso en desarrollo, abierto y no con-

clusivo, aunque sugerente en cuanto a una agenda temática. Las elaboraciones contenidas hacen eco tanto de la perspectiva que orienta su reflexión, como del trabajo colectivo y de las ponencias presentadas por el grupo de coordinadores con ocasión de las directrices temáticas propuestas. El presente artículo se divide en tres partes:

En la primera, titulada Bogotá como Ciudad Educadora: ihacia un nuevo modelo para pensar la escuela y la educación?, se hace una introducción a la problematización de "la idea y del modelo de Ciudad Educadora, llama la atención sobre la importancia de abordar los problemas contemporáneos de la pedagogía y de la política educativa desde una óptica anclada en la historicidad del saber pedagógico. Sea esta una invitación de carácter genealógico a ponderar lo que hoy se nos muestra como un horizonte deseable o un plan de acción presente.

En la segunda, se hace una presentación sucinta del Seminario de coordinadores y se retoman algunos de sus aportes conceptuales en torno a la relación entre 
Ciudad Educadora y tres directrices temáticas que orientaron la discusión durante el segundo semestre de 2005 , teniendo en cuenta la pluralidad y la diversidad de miradas. Estas contribuciones se han agrupado bajo el titulo Aproximación conceptual a la noción de Ciudad Educadora.

En la tercera, titulada Retos e implicaciones de la Ciudad Educadora en el contexto de Bogotá, se incluyen sugerencias y "mensajes de advertencia", surgidos en las sesiones y en las ponencias de los coordinadores.

\section{Bogotá como Ciudad Educadora: ¿hacia un nuevo modelo para pensar la escuela y la educación?}

Las intervenciones durante las sesiones del Seminario de coordinadores 2005, han oscilado entre el optimismo y la cautela con respecto a un modelo educativo alternativo para la ciudad. Optimismo, en tanto se resalta la potencia de abrir la escuela a la ciudad desde una validación de aquélla como espacio para la formación ciudadana y construcción de nuevas subjetividades. Optimismo, en tanto que la actual coyuntura invita a un replanteamiento de la forma de ser maestros y de ser escuela, generando condiciones para su desarrollo. Paralelo a este optimismo, se han planteado posiciones de cautela, que señalan la importancia de conocer los condicionamientos en los cuales está inmersa la escuela y el maestro; cautela, con respecto a imágenes mesiánicas de la acción educativa, cautela con la aplica- ción de planteamientos y estrategias surgidas en otras latitudes.

Entre las diversas posiciones que gravitan entre el optimismo y la cautela, surge la necesidad de establecer unos mínimos conceptuales entre lo pedagógico y lo político, entre la Cátedra de Pedagogía, el discurso de la educación pública y la organización política, de resaltar los peligros de avalar determinado modelo educativo, de desembocar en proyectos asistencialistas -dadas las prioridades hacia ciertos grupos poblacionales-, o de circunscribir el trabajo pedagógico a una mera curricularización de la ciudad. Por ello el Seminario ha tenido ante sí el reto de delimitar los conceptos que fundamentan la Cátedra de Pedagogía, y de abordar críticamente la idea y el modelo de Ciudad Educadora.

El propósito de conocer y de analizar las experiencias educadoras de otras latitudes en el ámbito de la Cátedra de Pedagogía, no ha sido otro que el de avanzar en una problematización del sentido de la escuela y del papel del maestro hoy, acentuando el carácter político y comunicativo de la mediación pedagógica y llamando la atención sobre las posibilidades de la relación escuela - ciudad. En esta discusión y otras que se planteen en el ámbito de la ciudad, no podemos perder de vista el horizonte conceptual de la pedagogía, o de la pedagogía como campo de batalla, valga decir, campo de acción política que se libra al interior de las escuelas, y en donde la relación, o mejor, la tensión escuela - ciudad, brinda un nuevo escenario. 
Pero además de apreciar experiencias actuales, también es importante conocer movimientos en nuestro país cuyo común denominador ha sido la educación. Me refiero al generado durante las décadas del veinte y del treinta del siglo XX, como parte de un movimiento mundial relacionado con la Escuela Activa, y una tendencia naciente que abogaba por la dignificación del magisterio y la educación pública, como objeto de acción gubernamental que cifró en la escuela y su acción extensiva gran parte del proyecto de construcción de la nación.

Si bien la orientación fundamental de los años treinta se dirige al establecimiento de un nuevo orden social, dimensionando desde una óptica diferente el problema del campo y la ciudad, del sector rural y del urbano, de la propiedad privada y la función social de la tierra, de los derechos individuales y colectivos en relación con los fines sociales del Estado y la propiedad individual, será la educación el ámbito desde y en torno al cual convergen, se sustentan y se adecuan las acciones políticas y económicas que alientan los nuevos designios y orientaciones de la época; también desde allí se desplegarán puntos de resistencia a la implementación de aquéllas. En uno y en otro caso, lo que se pone de presente durante la década del treinta es la educación como objeto de utilidad pública y de interés social: principio de buen gobierno, clave del progreso, instrumento político para la construcción y consolidación de la idea de nacionalidad. Las décadas del veinte y del treinta delimitan, para la his- toria del saber pedagógico, una serie amplia de cambios y transformaciones, desplazamientos y redefiniciones que irían a desembocar en ese gran movimiento cultural que en la época se conoció como la "Reforma Educacionista". En el proceso que da cuenta de su configuración intervinieron por lo menos cuatro elementos:

1. Restauración fisiológica y espiritual de la población.

2. Necesidad, propuesta y puesta en marcha de un proyecto de nacionalidad.

3. Adecuación, implementación y funcionamiento de un "aparataje técnico-administrativo", como un mecanismo hacia la modernización de los componentes de la vida nacional ${ }^{2}$.

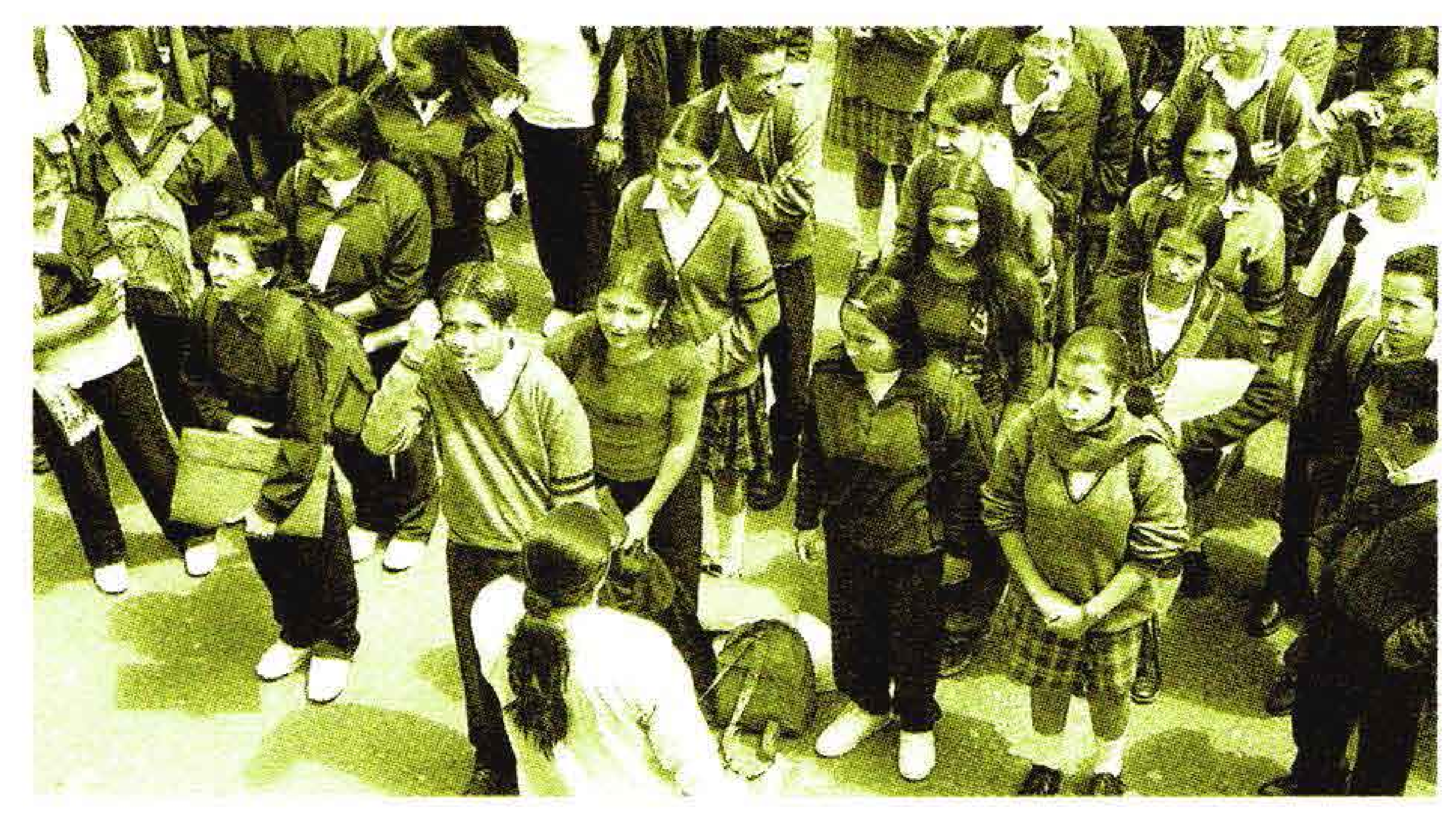

2 Véase, Quiceno, Humberto. "Pedagogía católica y Escuela Activa en Colombia”, Bogotá, Foro Nacional por Colombia, 1988. 
4. Crítica y reforma de los métodos de enseñanza, en una doble dimensión: como instrucción y como formación.

La composición geométrica que nos sugiere estos cuatro elementos constitutivos, se haya suspendida en una trama más amplia, que a su vez se encuentra atravesada y sustentada por tres ejes enunciativos clave dentro del período que corre desde la terminación de la Guerra de los Mil Días, y que en alguna medida todavía hoy halla sus prolongaciones. Los ejes enunciativos a los que me refiero son: Alma nacional, Progreso y Educación pública, coextensivos entre sí, pero con particularidades en cada caso. La Reforma Educacionista buscaba un cambio social apoyándose en las prácticas de escolarización y en la función extensiva de la escuela ${ }^{3}$. En ese entonces se trataba de emprender grandes realizaciones, que en palabras del ministro Luis López de Mesa, dejarían a

\footnotetext{
La década del treinta será testigo de una explosión institucional, en la doble perspectiva sobre la que nos llama la atención Aline Helg: de una parte, hacia la formación de las elites, y de otra, hacia la civilización del pueblo. Explosión institucional que se debate entre dos polos extremos cuyos casos más ilustrativos se hallarán representados de un lado, por el Gimnasio Moderno, el Instituto Técnico Central, el Instituto Pedagógico Nacional, la Facultad de Ciencias de la Educación (1932), la Escuela Normal Superior (1936), el Instituto Nacional de Educación Física (1937) y, de otro, por las Normales Rurales (1935), las Colonias de Vacaciones (1937), los Patronatos Escolares (1940), las Cooperativas, Granjas y Restaurantes Escolares, y las múltiples reglamentaciones en torno a la denominada "escuela complementaria", extensión a la escuela primaria de las funciones propias de una escuela de artes y oficios.
}

un lado aquellas acciones aisladas y focalizadas, para asumirlas dentro de un sentimiento nacional desde donde era "... indeclinable pensar en grandes magnitudes, no en ensayos de juguetería pedagógica..." .

Esta breve referencia llama la atención sobre un aspecto de vital importancia a la hora de ponderar propuestas como la de Ciudad Educadora: no perder de vista la historicidad de nuestra escuela, de nuestros maestros, de nuestra ciudad, y de acontecimientos y prácticas de saber tan significativos como educación y pedagogía en nuestro medio. No es gratuito entonces, el título de este trabajo en relación con el libro de Rafael Bernal Jiménez, uno de los gestores de la Reforma Educacionista, que nos hace afirmar hoy, parafraseándolo con igual vehemencia pero con la suficiente cautela: La Ciudad Educadora: ... he ahi el problema ${ }^{5}$. Miremos ahora la aproximación conceptual, todavía abierta y en construcción, que desde el Seminario de coordinadores hemos hecho de esta idea y modelo.

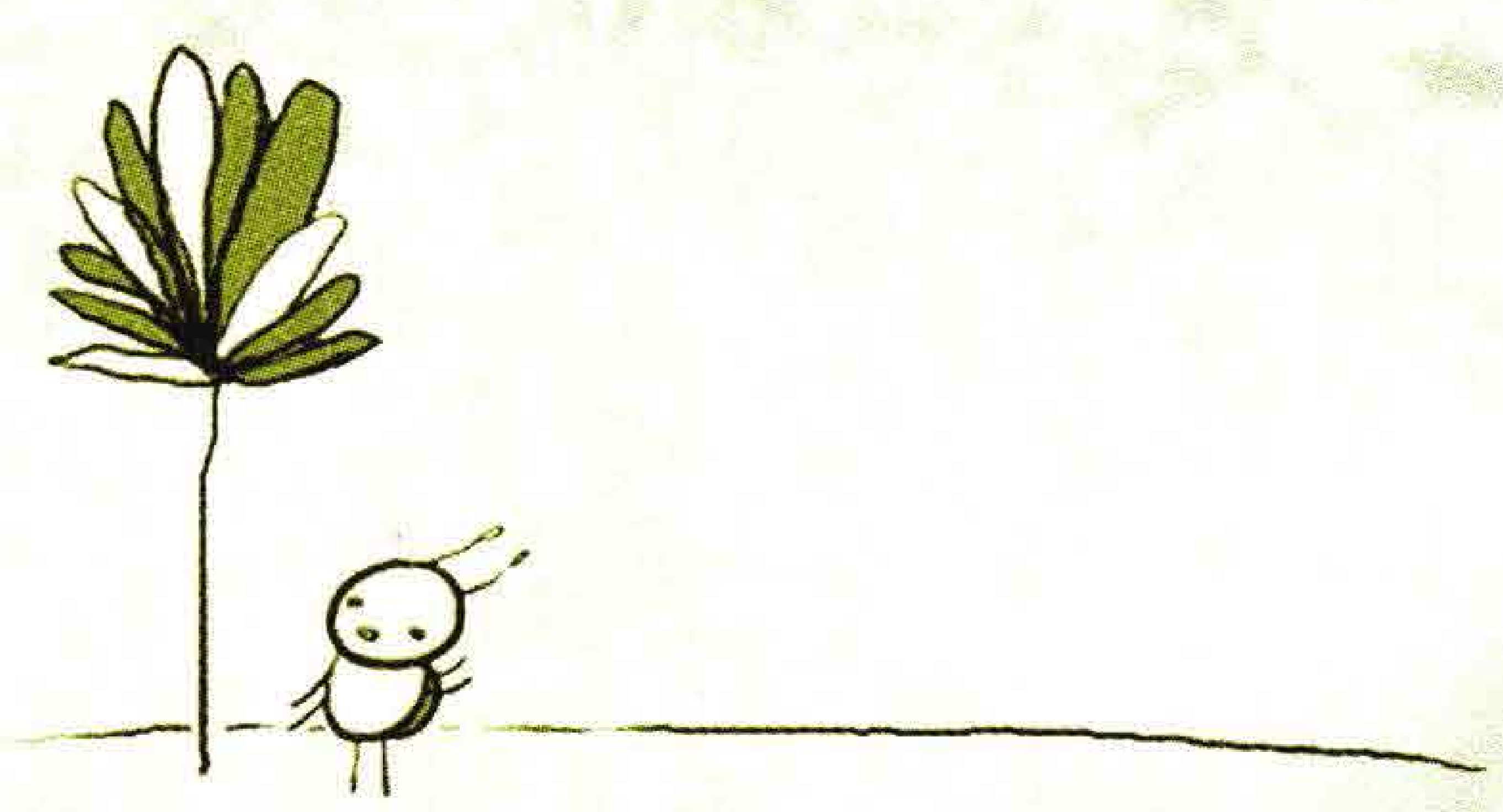

4 López de Mesa, Luis. "Tres Instituciones Pedagógicas Colombianas", en: Revista de las Indias, Vol. II, № 7, Bogotá, Ministerio de Educación Nacional, 1937, p. 52.

5 El texto al que aludo es La educación, he ahí el problema, de Rafael Bernal Jiménez, Bogotá, Ministerio de Educación Nacional, 1949. 


\section{Aproximación conceptual a la noción de Cludad Educadora}

Con ocasión de la Cátedra de Pedagogía 2005, centrada en el reconocimiento de experiencias de ciudades educadoras, el Seminario de coordinadores buscó explicitar, desde un ámbito académico y como una contribución a las instancias que fijan las directrices y lineamientos de política educativa, un conjunto de aspectos clave sobre los cuales sería posible aportar a la conceptualización de la relación escuela-ciudad. Como ámbito académico y encuentro de pares, la problematización se conjugó con la tematización de la discusión, en la cual se abordaron desde diferentes visiones los aportes y alcances de la idea y del modelo de Ciudad Educadora en el contexto de nuestra capital. En este sentido, esta aproximación conceptual buscó entrar en consonancia con las directrices de política pública desde la experiencia propia en el contexto de la localidad, teniendo como referentes el trabajo desarrollado con los maestros, las experiencias inscritas en el movimiento de ciudades educadoras y la perspectiva del colectivo de coordinadores ${ }^{6}$.

El seminario, de carácter académico, se desarrolló con base en unas preguntas directrices que orientaron la discusión, y sobre las cuales se elaboraron los

\footnotetext{
6 Los propósitos del Seminario de coordinadores se encuentran consignados en tres ítems ya anunciados en documentos programáticos del IDEP y de la SED, en los cuales encuentra sustento y pertinencia: 1) Identificar líneas de discusión que complejicen la reflexión sobre la relación escuela-ciudad; 2) Construir colectivamente desde el debate horizontes de comprensión de la relación escuela - ciudad; 3) Aportar colectivamente al desarrollo metodológico y conceptual del trabajo local en relación con el tema escuela-ciudad.
}

protocolos, considerados no sólo como un anexo de memoria sino como un referente. A su vez, las directrices temáticas propuestas surgieron como parte de las discusiones de los participantes, proponiendo un agrupamiento según ciertos tópicos y a partir de relaciones que, eventualmente, pueden articular diferentes posiciones y perspectivas sobre los cuales el Seminario buscaba avanzar en su conceptualización. Fueron tres las directrices temáticas propuestas:

a. Ciudad Educadora, desde la relación educación, ciudad y comunicación.

b. Ciudad Educadora, desde la relación entre lo gubernamental, la cultura política y la formación ciudadana.

c. Ciudad Educadora, desde la relación pedagogía, ciudad y escuela?

En el esbozo de estas tres temáticas, se encuentra un abanico in̨teresante de tópicos y relaciones que es necesario fortalecer en su conceptualización y articular en la composición estratégica que supone la inteligencia de las acciones interinstitucionales. La Ciudad Educadora no resuelve el problema de la escuela, ni la escuela puede asumir sola la responsabilidad de los designios de la Ciudad Educadora. Ni qué decir del maestro. Esto quiere decir, si seguimos los argumentos presentados en el Seminario, que la

\footnotetext{
7 Véase Cátedra de Pedagogía. Seminarios de coordinadores, Protocolo 5, Bogotá: SED-IDEP, 2005.
} 


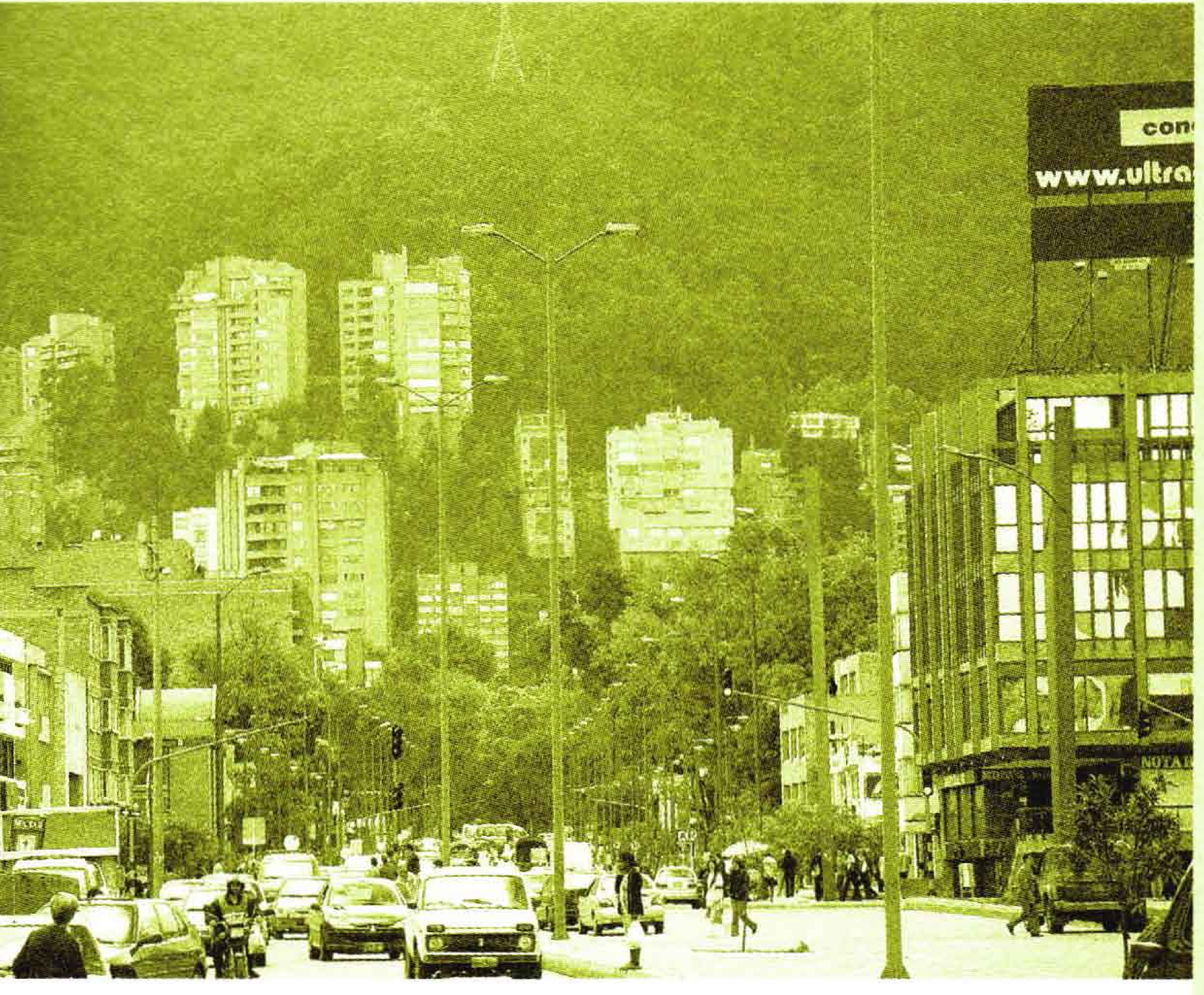

"tarea" es mucho más compleja, rica y no por ello menos riesgosa en las condiciones y contradicciones en las cuales se desenvuelve una metrópoli como Bogotá. Como veremos en la última parte de este artículo, la opción abierta desde la idea y el modelo de Ciudad Educadora para repensar las relaciones entre escuela y ciudad, medios de comunicación y ciudad, cultura y formación ciudadana, tiene varias implicaciones y retos. Lo que interesa a continuación es mostrar de manera global, algunos de los aportes a esta discusión desde el Seminario.

\section{Ciudad Educadora, desde la relación educación, ciudad y comunicación}

Una máxima define esta relación: la Ciudad Educadora es un hecho comunicativo, por tanto debe inscribirse y leerse desde modelos comunicativos. Las diferentes experiencias de ciudades educadoras presen- tadas ilustran con mayor o menor detalle esta condición, que parte del significado esencialmente comunicativo de la ciudad y de la educación, del valor de una y de otra como experiencias sociales y de lo social como hilos de comunicación (Cubides) o interacciones comunicativas más allá de los medios (Vásquez). Desde esta óptica es evidente la mediatización de la sociedad, sus implicaciones en la ciudad y la escuela, los consecuentes descentramientos tanto en las formas de relacionarse como en los procesos cognitivos, y la relación de los sujetos con el conocimiento, las intencionalidades de homogenización pero también los efectos de fragmentación a los que se ve enfrentada la construcción de subjetividades en el mundo contemporáneo. Los aportes indican la importancia de conocer y de hacer seguimiento a las incidencias que estos modelos comunicativos -paradigmas informacional y dialógicotienen en la producción y en la apropiación de proyectos de ciudad educadora, en los usos y maneras de habitar la ciudad, en tanto mediaciones culturales.

Esta es una aproximación a la relación que desde la perspectiva del Seminario de coordinadores sigue abierta para desarrollos posteriores a la luz del trabajo local y de las apropiaciones generadas en diversos grupos de maestros. Por ahora, es importante señalar algunos aspectos que invitan a pensar la $\mathrm{Ciu}$ dad Educadora desde la relación educación, ciudad y comunicación como:

a. Una opción que permite aguzar la mirada sobre las mediaciones implícitas en la 
acción educativa y la oportunidad de asumir la ciudad como texto a interpretar desde múltiples actores;

b. Una alternativa de análisis que asume lo pedagógico y las prácticas pedagógicas en tanto acción comunicativa inscrita en un juego de códigos y roles;

c. Una estrategia de descentramiento de la política de las instancias formales y jerárquicas, para asumirlas en un terreno de transacciones y apropiaciones de los múltiples sujetos que intervienen en el acto educativo;

d. Una invitación a la comprensión y análisis de las relaciones entre ciudad, educación y medios masivos de comunicación;

e. Un reconocimiento de las perspectivas procedentes de la semiología y la antropología que buscan repensar el papel de la función educativa.

\section{Ciudad Educadora, desde la relación entre lo gubernamental, la cultura, la política y la formación ciudadana}

En primera instancia, se advierte aquí la connotación política de la educación y la ciudad, conjugadas en la propuesta de Ciudad Educadora, ambas como esferas públicas en las que el ejercicio de la ciudadanía es posible y deseable. Desde allí se tejen pre-

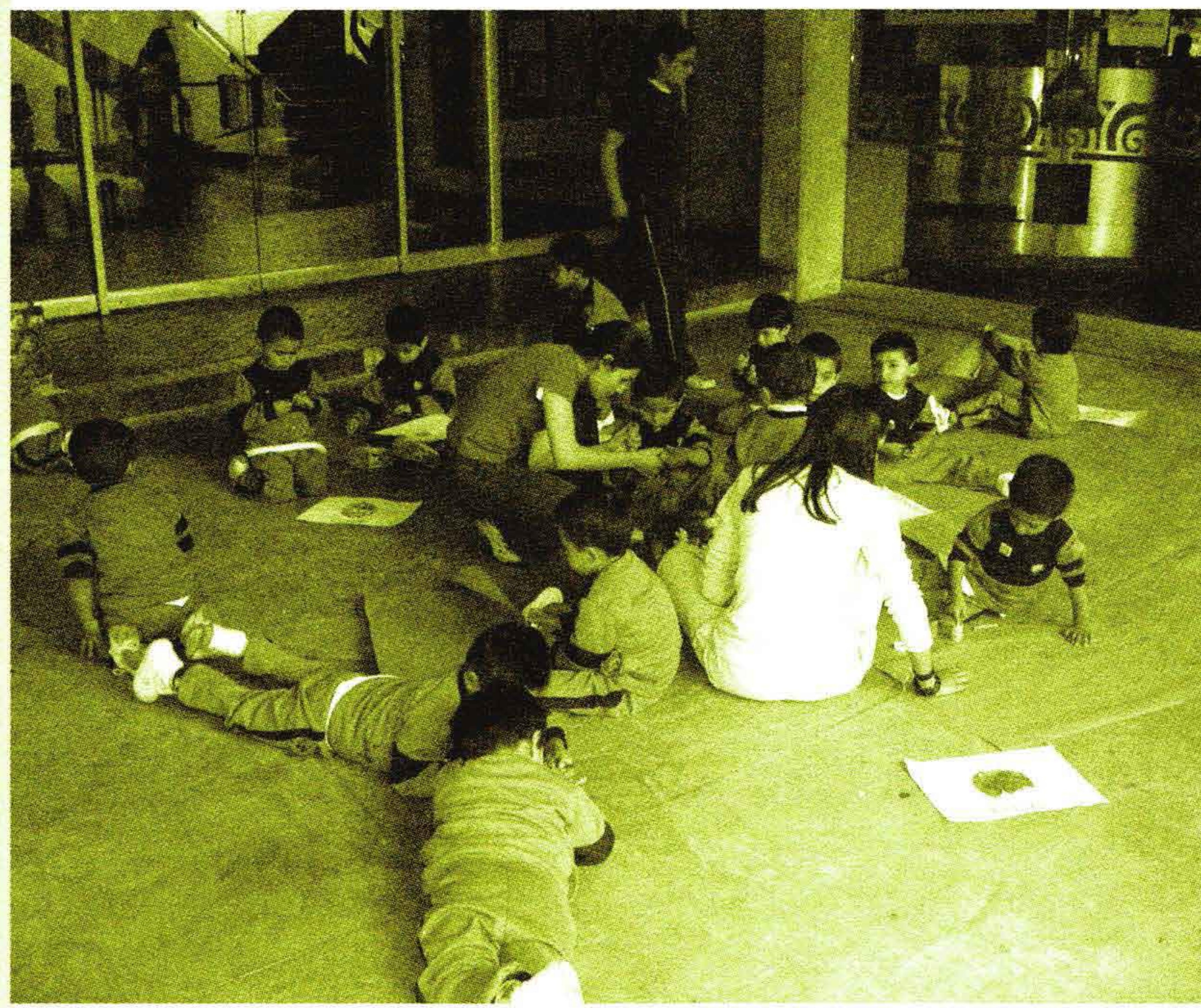

guntas y se abren planos de análisis que gravitan entre los aportes de la cultura política y del discurso en torno a los Derechos humanos y estrategias que tienen como objeto la reflexión sobre la ciudadanía y sus variantes en una contexto contradictorio, caracterizado por relaciones de fuerza cuyo común denominador es la exclusión, el marginamiento, la disolución, la fragmentación, el desarraigo y la deshumanización (Pinilla, Salazar). Esta perspectiva permite visualizar las tensiones entre la idealización del modelo de Ciudad Educadora y su puesta en escena, en condiciones de existencia concretas de los habitantes de la ciudad en donde el problema de la inclu- 


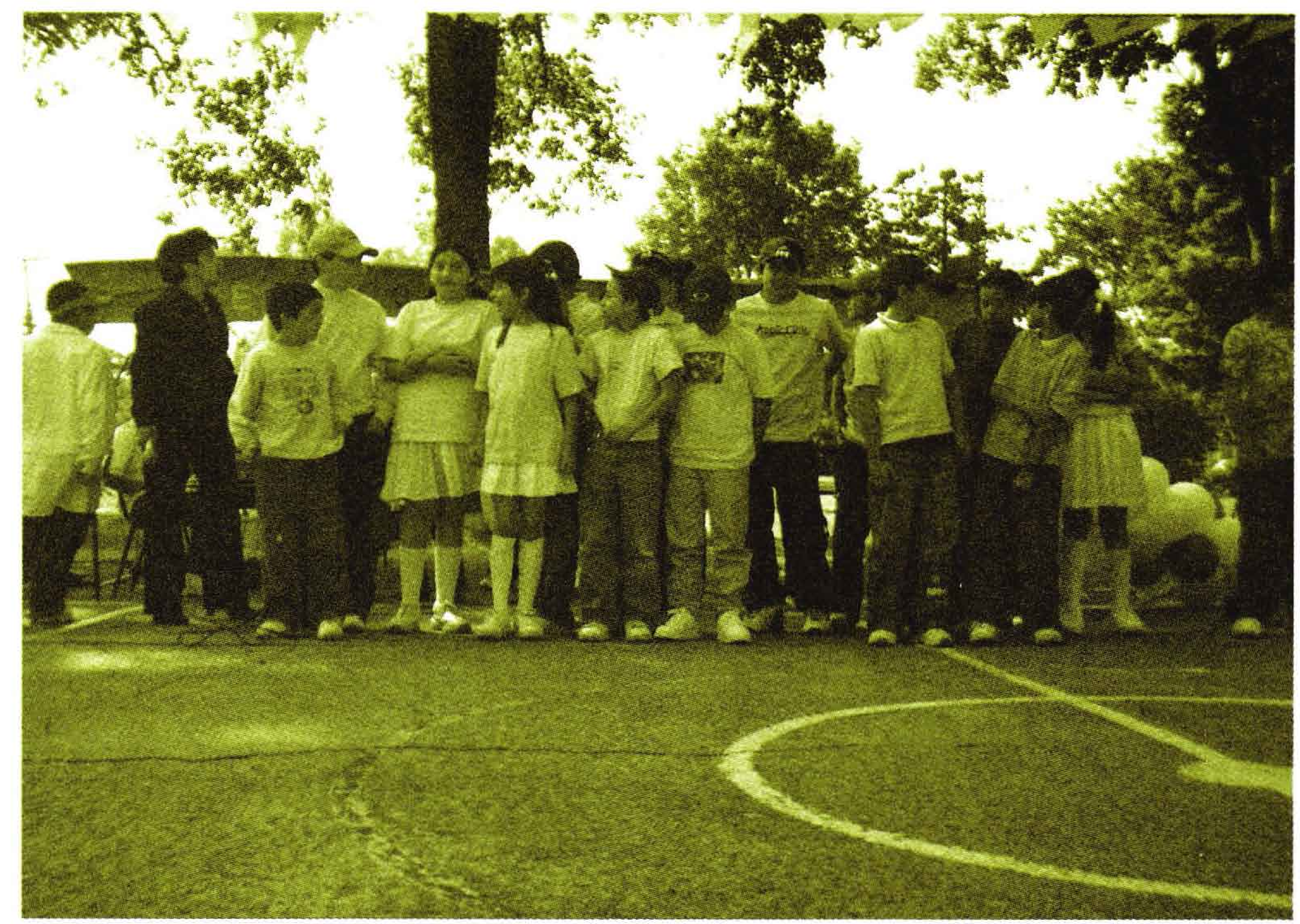

sión, la participación y la construcción de ciudadanía son retos vigentes, poniendo a su vez en evidencia el papel de la escuela, de los maestros y de las políticas públicas.

Estas acotaciones a la dimensión política sugieren varios aspectos fundamentales a la hora de problematizar la noción de Ciudad Educadora desde la relación entre lo gubernamental, la cultura política y la formación ciudadana, toda vez que nos invita a asumirla como,

a. Un modelo para orientar las políticas públicas relacionadas con la educación y con el gobierno de la ciudad;

b. Una posibilidad para la afirmación social de los sujetos sociales, y una oportunidad para la participación y la movilización de diferentes actores y grupos en consonancia con el derecho a la educación;

c. Un replanteamiento de la formación ciudadana, tanto en la promoción como en los efectos que implica la afirmación de la función social de la escuela;

d. Una alternativa para desplegar estrategias y mecanismos que liguen la educación y la escuela a los imperativos de inclusión social;

e. Un reconocimiento de una perspectiva que aboga por la dimensión política y la movilización social en donde la pedagogía crítica aporta a la relación ciudad escuela. 


\section{c i u d a d \\ e d u c a d o \\ Ciudad Educadora, desde la relación pedagogía, ciudad y escuela}

Esta relación toca directamente el sentido de la escuela en el territorio, la ciudad como escenario educativo y la pertinencia de una mirada en clave pedagógica, valga decir, desde su horizonte conceptual, que atienda a las condiciones en las cuales se cumple su funcionamiento social, con ocasión de los procesos de institucionalización generados en las escuelas y del trabajo de los maestros y sus apropiaciones a la luz de su quehacer cotidiano unido a propuestas de alternativas de subjetivación. En las ponencias presentadas se advierten diferentes variantes y planos de análisis de esta relación. En una de ellas, se pone el acento en el papel de la escuela y la connotación politica del tema ciudad y comunidad en el trabajo de los maestros (Bryon); surge la pregunta por la pedagogía y su lugar en la escuela y la ciudad en íntima relación con las prácticas transformadoras, y la importancia de estas dos últimas como construcciones colectivas en las cuales es fundamental el reconocimiento del otro y el lugar de la diferencia (González). Por otro lado, se encuentran aquellas reflexiones que atan la noción ciudad - escuela a su dimensión histórica y cultura, a los cambios de composición de la ciudad con las posibilidades de abrir la escuela (Acosta, Salinas). Las opciones de tematización sugeridas por una de las ponencias a la luz de las implicaciones de los discursos y prácticas de la Ciudad Educadora teniendo como eje la relación ciudad - escuela, hizo explícita su pregunta por el maestro y las concepciones de pedagogía y educación que promueven, y fue sugestiva por cuanto evidenció el mo-

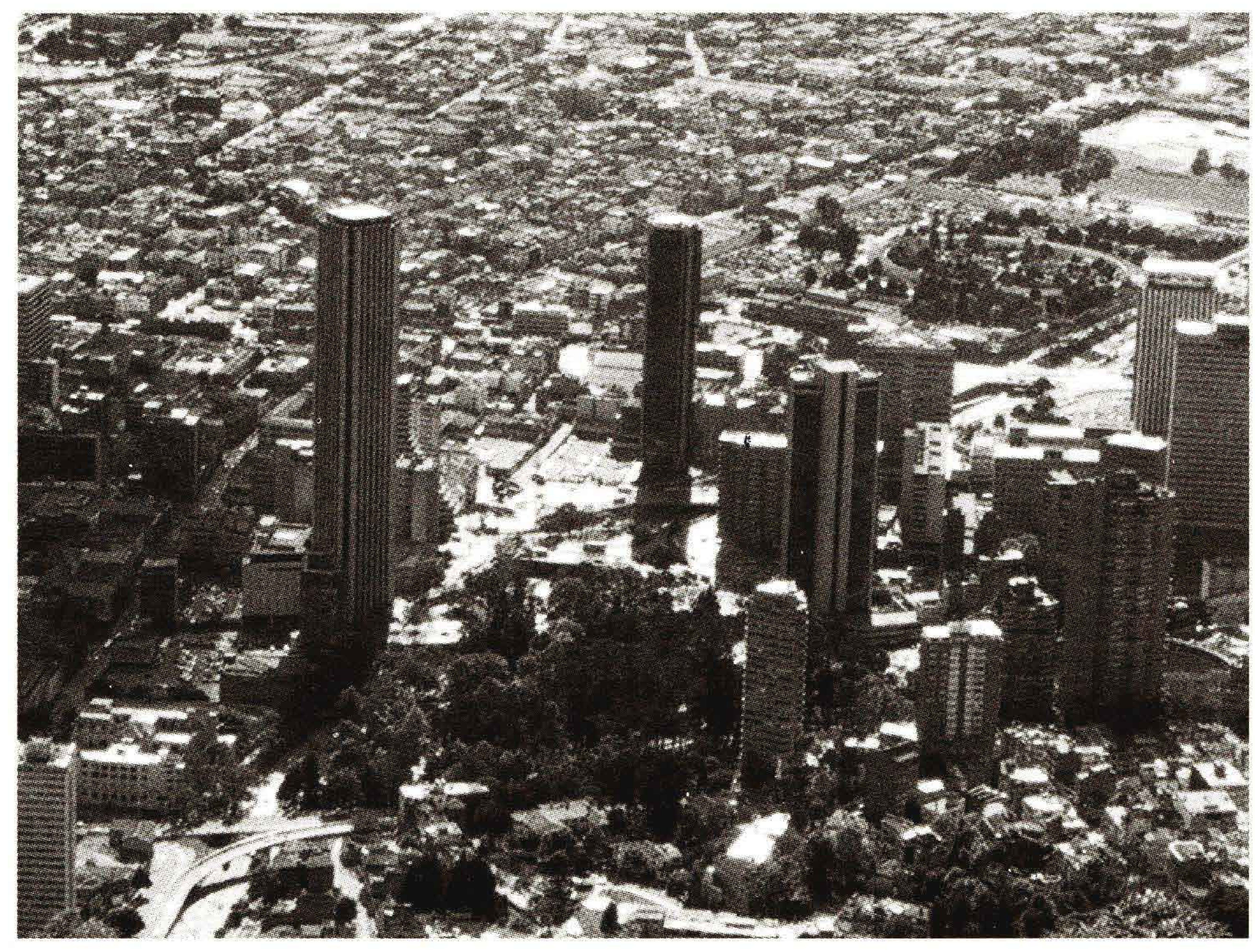


delo en el que se inscribe la experiencia de Bogotá dentro de la clasificación propuesta (Díaz) ${ }^{8}$.

Los aspectos arriba mencionados, además de mostrar la multiplicidad de miradas, indican la pertinencia de una pregunta que anude la noción de Ciudad Educadora a las relaciones pedagogía, ciudad y escuela, que como en los casos anteriores inviten a pensar esta relación como:

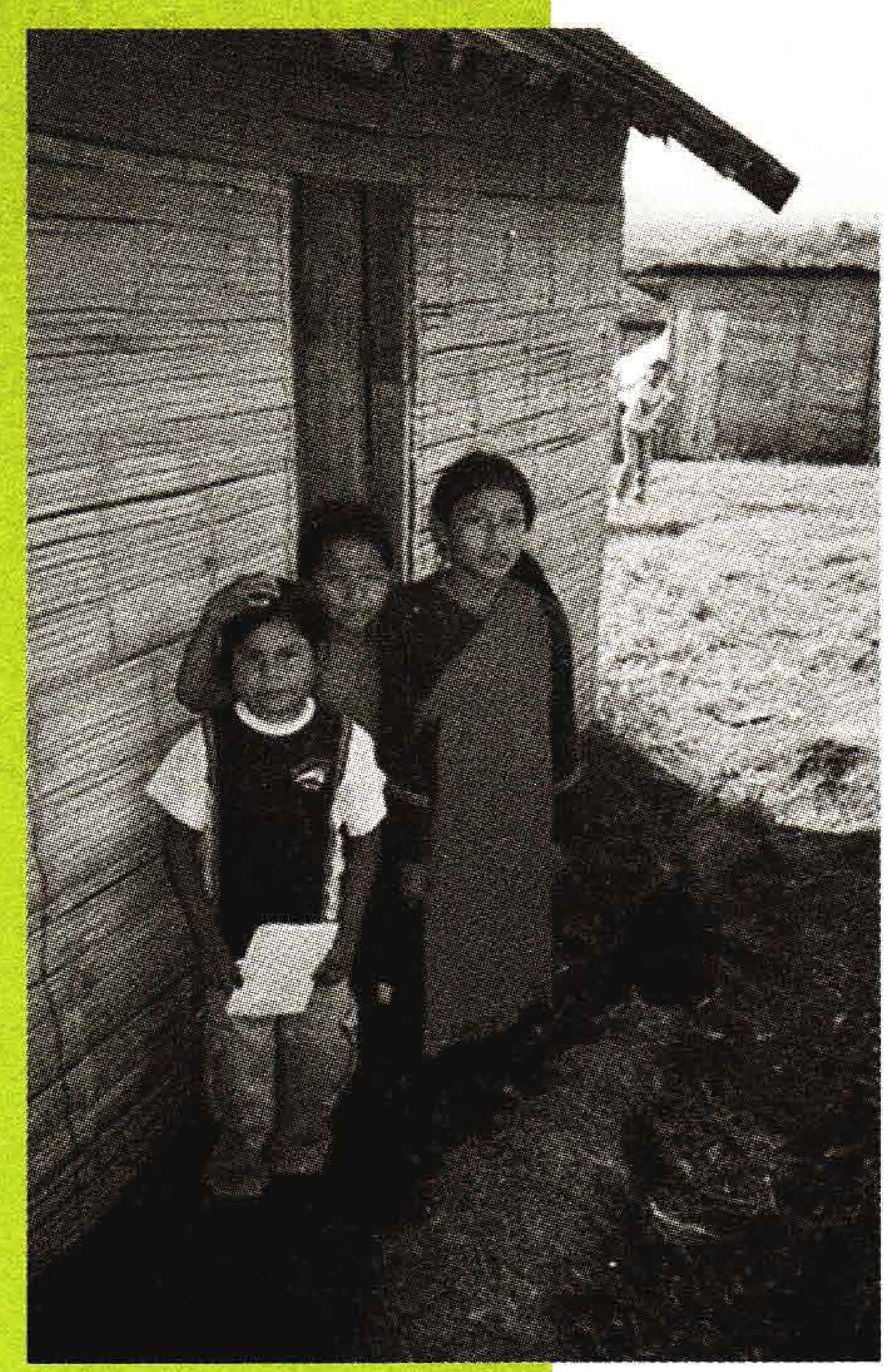

a. Una posibilidad de ligar la reflexión pedagógica a su contemporaneidad, lo cual impacta a la escuela, al maestro, y plantea su incursión en otras formas de hacer politica;

b. Una oportunidad para afectar el papel de maestros, estudiantes y de la escuela re-pensando la ciudad no solo como un tema del plan de estudios o de ejercicio lúdico;

c. Un nuevo reto a los maestros, padres y personal administrativo;

d. La reafirmación de la escuela en tanto territorio y generadora de nuevas territorialidades, tanto en los saberes como en las nuevas disposiciones de sujetos y su interacción con otros actores;

e. Una forma de abordar los saberes que circulan por la escuela y que la constituyen;

f. Un reconocimiento de la historicidad de la pedagogía, su horizonte conceptual, la facultad exten-

8 Al respecto, Olga Cecilia Díaz alude a tres tendencias discursivas: Ciudad Educadora como escuela para la sociedad; Ciudad Educadora como escuela para la inclusión y la humanización; Ciudad Educadora como escuela del sujeto, siguiendo algunos planteamientos de Touraine. 
siva de la escuela y la alianza estratégica entre pedagogía y ciudad.

\section{Retos de la Ciudad Educadora en el contexto de Bogotá}

Varios retos e implicaciones plantea el modelo de Ciudad Educadora en el contexto de una ciudad como Bogotá. A continuación presentaré, de manera esquemática, algunas reflexiones generadas en el Seminario de coordinadores.

- Promover diversos planos de análisis sobre la experiencia generada con ocasión de las relaciones establecidas entre ciudad y escuela, y los desarrollos pertinentes para avanzar en un proceso de Ciudad Educadora. Quizás esta sea una de las prioridades no sólo de cara a la conceptualización y la fundamentación de un proyecto político, sino de la necesaria apropiación y participación de maestros, comunidades, gremios, instancias de gobierno distrital, universidades, empresas y entidades privadas. Los planteamientos de Alicia Cabezudo, Pilar Figueres y María Aparecida, entre otros, corresponden a acciones programáticas deseables que requieren adecuarse a las condiciones de Bogotá.
- Es interesante resaltar aquellas sugerencias que ubican en el centro a la gente, a los habitantes de la ciudad, tanto en su dimensión afectiva como en propuestas que reclaman como punto de partida la generación de nuevos procesos de subjetivación. En este mismo sentido, pero de manera específica, se resalta la importancia de fortalecer y de promover alternativas de formación y participación para el maestro, desde su saber y reconocimiento. Si bien la Ciudad Educadora

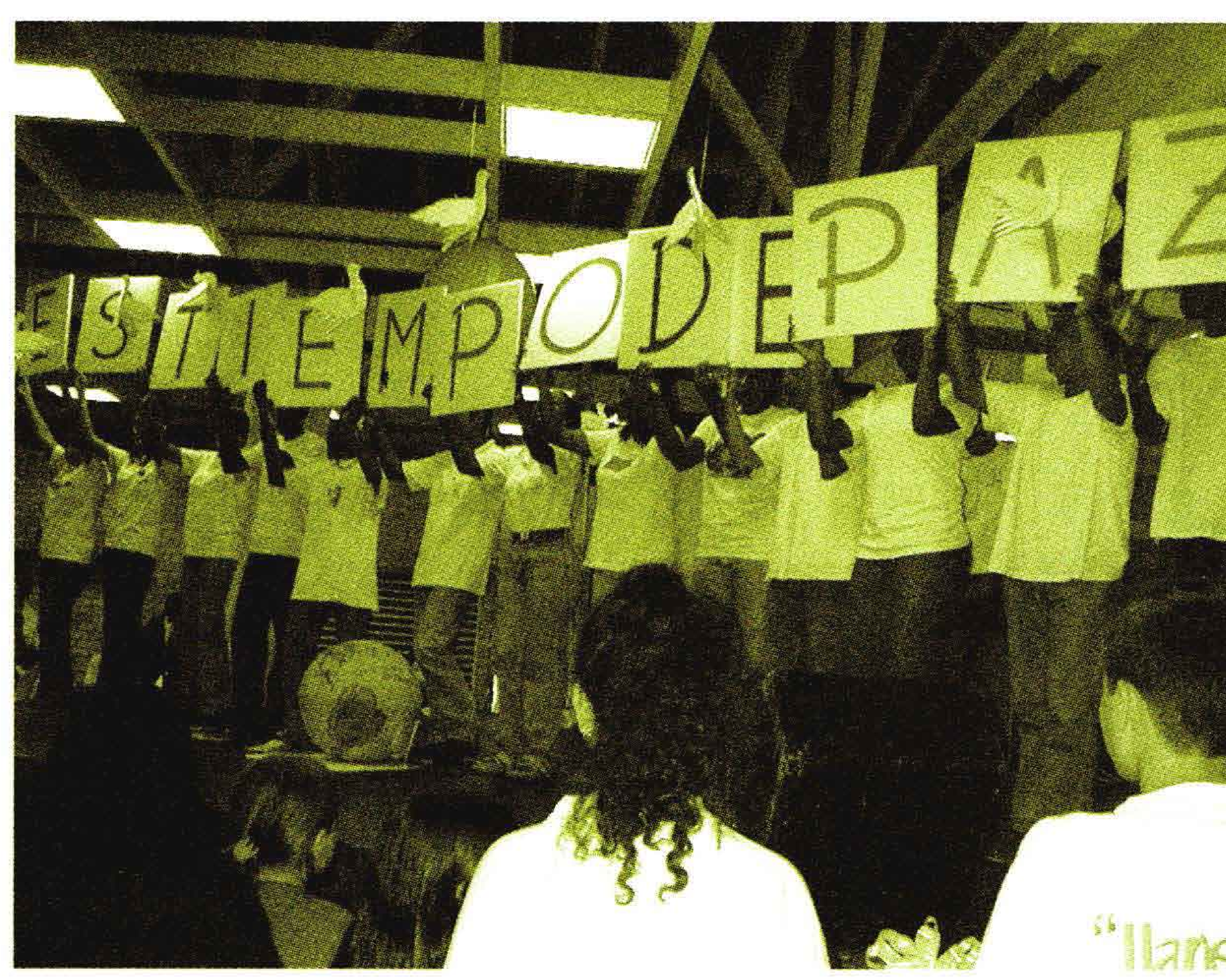


corre el riesgo permanente de quedarse en retórica o de ser asumido solo desde visiones instrumentales, si no existen avances concretos en la transformación de la cultura política que prevalece en la ciudad y repercute en la escuela.

- La incursión y afianzamiento de la relación escuela - ciudad, y las modalidades de acción estratégica que supone la Ciudad Educadora, implican conectar de manera urgente la pedagogía con una política pública intersectorial ${ }^{9}$. Queda como un reto de la Secretaría de Educación y del IDEP en su agenda de los próximos años, afianzar su liderazgo y su capacidad de convocatoria con otras secretarías y en general con el sistema de gobierno de la ciudad, en lo que tiene que ver con el desarrollo de la Ciudad Educadora y la definición de las políticas públicas.

- La ciudad requiere de un programa permanente de reconocimiento de su densidad y diversidad pedagógica y cultural. Ello implica continuar el diálogo y el intercambio local de experiencias pedagógicas unido a otras experiencias de ciudades educadoras, una relación necesaria hacia el fortalecimiento y despliegue de la experiencia de Bogotá. Repensar y resignificar la relación escuela - ciudad, es una de las condiciones más importantes para afianzar y desplegar esta alianza.

\section{Bibliografía

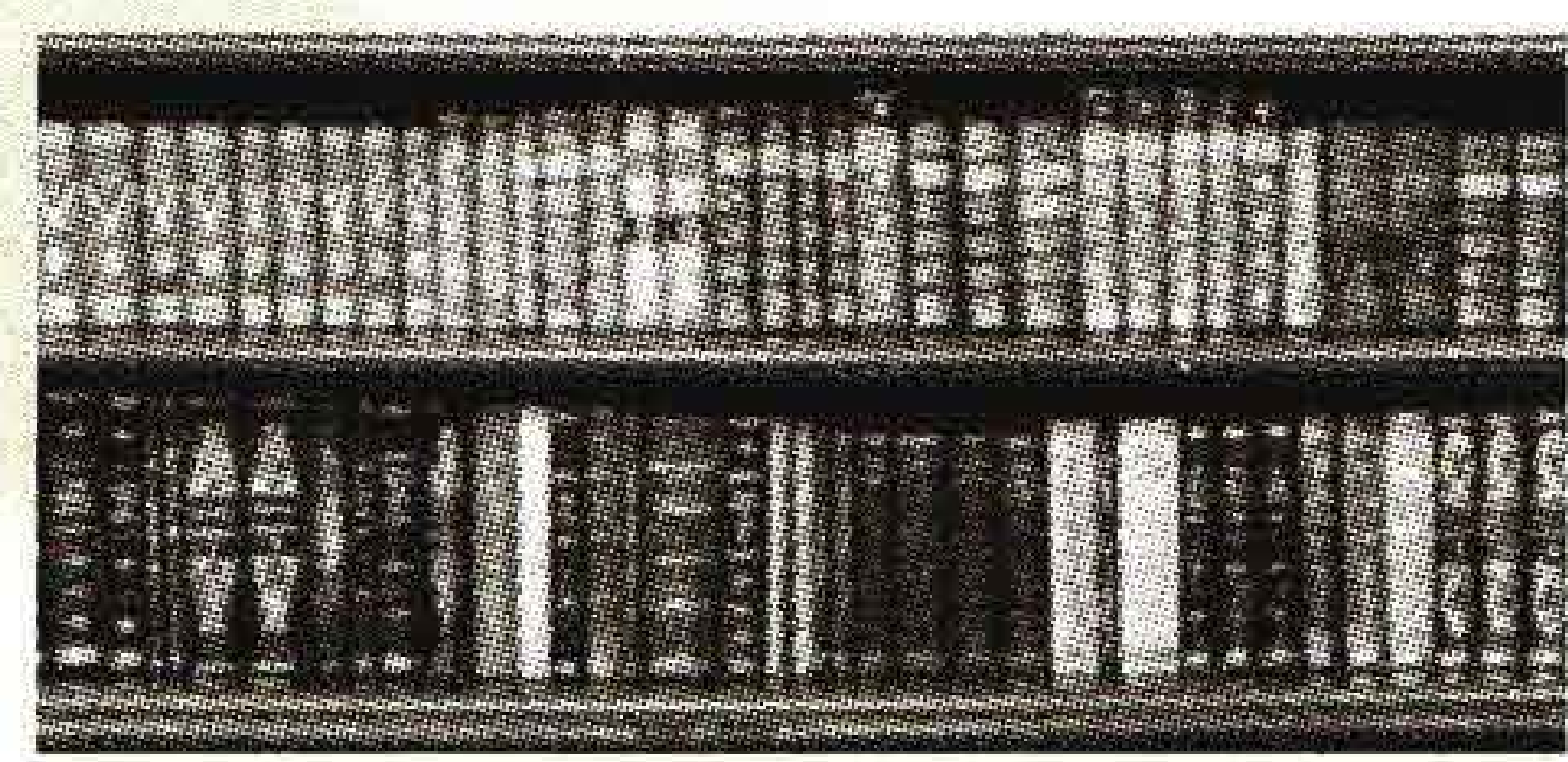

Acosta Jiménez, Wilson y Tarquino G., Ingrid Mayerli. Elementos para reflexionar las implicaciones sobre las relaciones ciudad educadora, escuela, pedagogía y maestro. Ponencia presentada al Simposio Reflexiones

9 Al respecto, los aportes de Alicia Cabezudo en la Cátedra 2005 y los planteamientos de Moacir Gadotti con respecto a la experiencia de Sao Paulo (Brasil), son referentes fundamentales para visualizar un programa a mediano plazo. sobre Ciudades Educadores. Bogotá: SEDIDEP, 10 de octubre 2005.

Bernal Jiménez, Rafael. La educación, he ahi el problema, Bogotá, Ministerio de Educación Nacional, 1949.

Bryon, Patricia y Gaona, Gustavo. Ciudad Educadora: Entre la realidad y la utopía. Ponencia presentada al Simposio Reflexiones sobre Ciudades Educadores. Bogotá: SED-IDEP, 10 de octubre de 2005. 
Cátedra de Pedagogía. Ponencias Conferencias Magistrales. 2005

Cátedra de Pedagogía. Seminario de Coordinadores. Protocolos. 2005.

Cubides, Humberto, Valderrama H., Carlos Eduardo, Durán, Armando y Valenzèela, Carlos E. Significado comunicativo de la ciudad educadora. Ponencia presentada al Simposio Reflexiones sobre Ciudades Educadores. Bogotá: SED-IDEP, 10 de octubre de 2005.

Díaz, Olga Cecilia y Valderrama, Camilo Andrés. Implicaciones de los discursos y prácticas de ciudad educadora. Ponencia presentada al Simposio Reflexiones sobre Ciudades Educadores. Bogotá: SED-IDEP, 10 de octubre 2005.

González Lara, Olga Maritza. Ciudad Educadora: Pedagogía, Escuela, Ciudad. Ponencia presentada al Simposio Reflexiones sobre Ciudades Educadores. Bogotá: SED-IDEP, 10 de octubre 2005.

Pinilla Díaz, Alexis, Nemojón, Jenny Liliana y Cárdenas Guillermo H. La ciudad incluyente: espacio de construcción de culturas políticas democráticas y ciudadanías radicales. Ponencia presentada al Simposio Reflexiones sobre
Ciudades Educadores. Bogotá: SED-IDEP, 10 de octubre 2005.

Salazar Montenegro, Irma y Mora, Rocío. Bogotá, Ciudad Educadora. Una paradoja o un proceso vital de desarrollo. Ponencia presentada al Simposio Reflexiones sobre Ciudades Educadores. Bogotá: SED-IDEP, 10 de octubre de 2005

Salinas Herrera, José Aladier. De la escuela a la ciudad. Ponencia presentada al Simposio Reflexiones sobre Ciudades Educadores. Bogotá: SED-IDEP, 10 de octubre 2005.

Vásquez Arrieta, Tomás y Bustamante B. Boris. Ciudad - escuela desde la perspectiva de comunicación-educación. Ponencia presentada al Simposio Reflexiones sobre Ciudades Educadores. Bogotá: SED-IDEP, 10 de octubre 2005. 